\title{
Retina blood vessel extraction based on Kirsch's template method
}

\author{
Nur Syazlin Zolkifli ${ }^{1}$, Ain Nazari ${ }^{2}$, Mohd Marzuki Mustafa ${ }^{3}$, Wan NurShazwani Wan Zakaria ${ }^{4}$, \\ Nor Surayahani Suriani ${ }^{5}$, Wan Nur Hafsha Wan Kairuddin ${ }^{6}$ \\ ${ }^{1}$ Faculty of Electrical and Electronic Engineering, Universiti Tun Hussein Onn Malaysia, Malaysia \\ ${ }^{2,4}$ Department of Mechatronic \& Robotic Engineering, Faculty of Electrical and Electronic Engineering, \\ Universiti Tun Hussein Onn Malaysia, Malaysia \\ ${ }^{3}$ Department of Electrical, Electronic \& System Engineering, Faculty of Engineering and Built Environment, \\ Universiti Kebangsaan Malaysia, Malaysia \\ ${ }^{5}$ Department of Computer Engineering, Faculty of Electrical and Electronic Engineering, \\ Universiti Tun Hussein Onn Malaysia, Malaysia \\ ${ }^{6}$ Department of Electronic Engineering, Faculty of Electrical and Electronic Engineering, \\ Universiti Tun Hussein Onn Malaysia, Malaysia
}

\begin{tabular}{l} 
Article Info \\
\hline Article history: \\
Received Sep 9, 2019 \\
Revised Oct 20, 2019 \\
Accepted Nov 5, 2019 \\
\hline
\end{tabular}

\section{Keywords:}

Extraction

Image morphology

Kirsch's template

Retinal blood vessel

\begin{abstract}
Analysis on the retina blood vessels from fundus images have been widely used in the medical community to detect the disorder condition in the blood vessels. An automated tracing of retina blood vessel can help to provide valuable computer-assisted diagnosis for the ophthalmic disorders. Thus, it helps to reduce the time for the ophthalmologist to analyses and diagnose the result of the fundus image of patient. The purpose of this research is to build an algorithm to trace the retina blood vessels. The method to be used in this research consist of two parts which are the pre-processing part and the feature extraction by using the Kirsch's template. Combining the pre-processing at the early stage and feature extraction at the next stage is applied to extract the edges of the blood vessels. The proposed algorithm was verified by using two online databases, DRIVE and HRF to validate the performance measures. Hence, proposed method is capable to extract the retina blood vessel and give the accuracy of 0.7917 , the sensitivity of 0.9077 and the specificity of 0.7215 . In conclusion, the extraction of the blood vessels is highly recommended as the early screening stage for the eye diseases beneficially
\end{abstract}

Copyright () 2020 Institute of Advanced Engineering and Science. All rights reserved.

\section{Corresponding Author:}

\section{Ain Nazari,}

Department of Mechatronic \& Robotic Engineering,

Faculty of Electrical and Electronic Engineering,

Universiti Tun Hussein Onn Malaysia, Johor, Malaysia

Email: ain@uthm.edu.my

\section{INTRODUCTION}

Retina is a thin layer of tissue that lines at the back of the eye on the inside and it purpose is to send signals to the brain for visual recognition. Retina blood vessel consist of arteries and veins which help to transport the blood throughout the eyes. It is one of the important elements in the eyes. But there can be a situation where the disorder in blood vessels can lead to diseases. Some of the example that can arise from retina blood vessel disorders are retinal vein occlusion, hypertensive retinopathy, central retinal artery occlusion, wet macular degeneration, diabetic retinopathy, ocular ischemic syndrome and others. According to [1] there are about one hundred and three patients were diagnosed with central retinal artery occlusion between January 2009 and December 2017. All this disease can lead to loss of vision blindness, and even stroke. As 
stated by [2] an early detection of subclinical diabetic retinopathy could contribute to the management and timely recognition for the patients before it gets worse.

Researchers from engineering, medical and others that related to the fields have studied and researched about the various symptoms and diseases that can arise from the retina. There are also other eyes disease that can arise from the other elements in the retina but in this research, it only focuses on the retina blood vessel. In this research, the fundus image from the online database is use as the element of the research. The online databases that will be evaluated are DRIVE [3] and HRF [4] databases.

This paper about to propose a method for retina blood vessel extraction to help the specialists in analyse, diagnose and give treatment to the patient with various retinal diseases [5]. To achieve this purpose, the extraction method needs to be accurate and reliable [6]. The proposed method uses the Kirsch template as the method for extracting the blood vessel. The Kirsch template is one of the various techniques that can be used to extract the retinal blood vessel. The previous research shows that plenty of researcher also used this method to segment the blood vessel [7-11]. This method also can be applied to detect the optic disk edges [12]. As stated by M. Joshita et al. [7], by using this template their proposed algorithm is successful and robust in extracting the retinal blood vessels accurately.

Research on the tracing of retina blood vessel have been widely utilized with various methods of segmentation being used. Some of the various methods are supervised and unsupervised learning [5, 13]. This method divides the data into groups based on their similarity measure such as the 'non-vessel' and 'vessel' group. Next, the matched filtering (MF) approach propose by Kolar et. Al [14, 15]. The 2D MF make use of the 2D masks and connection of local image areas. Five 2D filters were modelled according to typical blood vessel cross-sectional intensity profiles while another five different blood vessel widths were considered (thinnest to thickest). The image obtained from the preprocessing part is convolved with each of the five kernels, then is rotated to 12 orientations. The locally maximum response is selected for each pixel by the fused of resulting parametric images. Then, the image is threshold to acquire the binary map of the blood vessel tree.

Subsequently, a method using the top-hat multi-scale detection proposed by Ain Nazari et al. [16]. The origin of this method is proposed by Nguyen et al. [17] ] but is being improved in the pre-processing phase. This is because the previous method generates strong false vessel detection, and mostly in the area of the optic disk. Thus, with the improved method, it is designed to produces an enhanced input image that provides more blood vessels information and reduce false vessel response at the optic disk area. This method is also used by Yanli Hou [18] which propose the improved multiscale line detector to yield the blood vessel response. In addition, there also others method using the adaptive thresholding technique [19, 20], Bar-selective Combination of Shifted Filter Response (B-COSFIRE) [21, 22], Kirsch method [7-11] and others. The research is able to reduce the time for the opthalmologist to analyse and diagnose the result of the fundus image of patient. This research is important because eyes disease can lead to a loss of vision, thus it need to be detected and treated early before it gets worse.

The structure of the paper is presented as follows; Section 2 a sequence of explanation on the proposed research method. While, Section 3 discusses the results and analysis of the finding. Finally, a general conclusion is given in Section 4.

\section{RESEARCH METHOD}

\subsection{Overview}

There are two method conducted in this proposed algorithm which are the pre-processing method and the edge extraction using the Kirsch's template method. The proposed algorithms work on the extraction of the blood vessels from the retinal fundus image. Figure 1 shows the process of the proposed method.

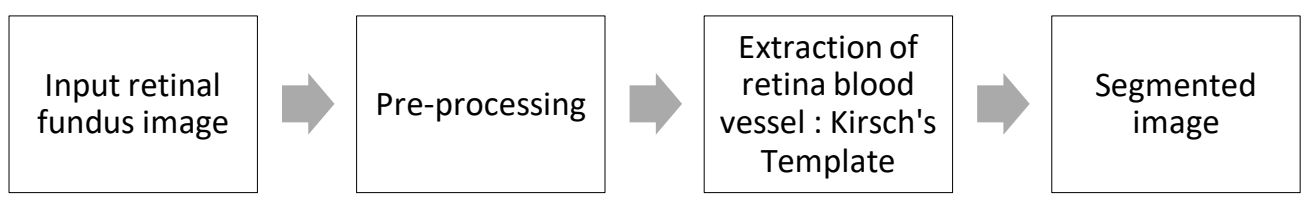

Figure 1. Block diagram for the proposed method

\subsection{Pre-Processing}

Color image usually have a lot of noise. In the case of the retinal fundus image the noise may come from the moment of capturing or when there are symptoms of diseases in the retina image of the patient. In this proposed method, it only extracts the retina blood vessel without considering any disease that unrelated to the 
retina blood vessel. Hence, the pre-processing process is importance to be conducted to remove the noise, or any unwanted artifacts and enhance the retina fundus image before undergoing further analysis stage [23].

In the pre-processing process, there are three methods being used: (1) Unsharp masking method (2) Contrast Limited Adaptive Histogram Equalization (CLAHE) (3) Gaussian filtering. Figure 2 (a and b) shows the fundus image from the online databases is use as the input for the system and the unsharp masking method is carry out to sharpen the image. The concept of the unsharp masking method is to blur the actual image and the next step is to subtract the blurred image from the actual image [24]. It focused on enhancing the edges and tiny details of the image. But there are drawbacks using this method which are the contrast in the darker area will appear heavier than the lighter area and it also enhances the noise. Yet, it can be handled by using further image enhancement technique. Next, using the Contrast Limited Adaptive Histogram Equalization (CLAHE) to enhance the contrast of the image as in Figure 2 (c). This method is used to improve the contrast and to reduce the noise amplification. CLAHE helps to avoid over accentuate the noise present in the image and limit the contrast. This method is most used in the pre-processing of the medical images. The final stage is the Gaussian filtering. Figure 2 (d) display the image after Gaussian filtering is conducted to eliminate the noise. The noise is added by using the denoising network and then is remove by the filtering process.

Lastly, the MSE and PSNR is calculated to measure the effectiveness of the method used. Mean Square Error (MSE) indicate the cumulative squared error between the pre-processed image and the original image, while PSNR represents the measure of the peak error.

$$
\begin{aligned}
& M S E=\frac{1}{m n} \sum_{i=0}^{m-1} \sum_{j=0}^{n-1}[I(i, j)-K(i, j)]^{2} \\
& P S N R=10 \log _{10}\left(\frac{M A X_{I}^{2}}{M S E}\right) \\
& =20 \log _{10}\left(\frac{M A X_{I}}{\sqrt{M S E}}\right)
\end{aligned}
$$

where, $M A X_{I}$ is the maximum possible pixel value of the image.

The method used by S. Badsha et al. [11] in the pre-processing part, are the method of highlighting the edges and conversion to grayscale and the needs to be filtered and histogram equalized because of the uneven noise. Proceed with the binarization and object classification. The result image from S. Badsha is shown in the Figure 2 (e). While, in this proposed method, image sharpening is added to improve the accuracy of the retina blood vessel extraction.

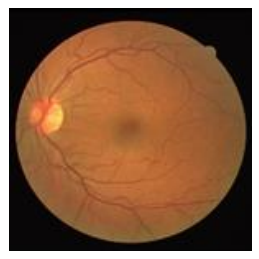

(a)

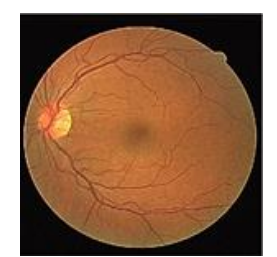

(b)

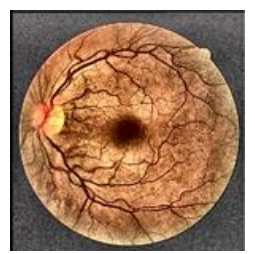

(c)

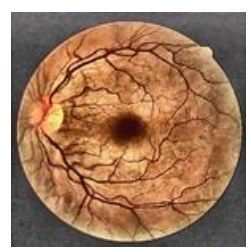

(d)

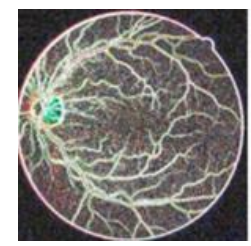

(e)

Figure 2. Pre-processed image (a) Input retina image (b) Sharpened retina image (c) CLAHE image (d) Gaussian filtered image (e) S. Badsha et al pre-processed image

\subsection{Blood Vessel Extraction Using Kirsch's Template}

The next step is the feature extraction where the blood vessel of the retina will be extracted. The method that will be used in this process is the Kirsch's template method. It is used to extract the blood vessels present in the retina and to enhance the edge of the blood vessels. Kirsch's template is a non-linear edge detection method that detect the maximum edge strength in a pre-determined direction [7]. Through edge the detection, the amount of data to be processed can be reduced, while the important structural properties in the image is still can be preserved [25].

After the noise has been removed by the filtering process in the earlier pre-processing, the blood vessel then can be extracted. Figure 3 (a) shows the used the Kirsch's template of size 3x3 in this proposed method. This process is to identify the pixel values in the image. The output of this method is the production of image consist of grey level pixels of value 0 and 255. The 0 -pixel value indicate the black pixel while the 255-pixel value indicate the white pixel. The brightness level of the neighboring pixels determined the edge information for the targeted pixel. The possibility to detect the edge in the image is based on the brightness levels difference present in the image. If there are no difference, so there is no edge detected [26]. 
The algorithm detects direction of the edge in all directions. There are eight direction that are possible to be use. The single mask is used and is rotated $45^{\circ}$ increments to include the 8 directions [12]. The template of the directions is in the form of matrices. The matrix has the information of the pixels and its surrounding neighbors. Out of the eight templates, the biggest will be treated as the output value and the edges will be extracted. Followed by the thresholding, it can be set and reset to obtain the most suitable edges needed.

$$
\begin{aligned}
& {\left[\begin{array}{ccc}
5 & -3 & -3 \\
5 & 0 & -3 \\
5 & -3 & -3
\end{array}\right]\left[\begin{array}{ccc}
-3 & -3 & -3 \\
5 & 0 & -3 \\
5 & 5 & -3
\end{array}\right]\left[\begin{array}{ccc}
-3 & -3 & -3 \\
-3 & 0 & -3 \\
5 & 5 & 5
\end{array}\right]\left[\begin{array}{ccc}
-3 & -3 & -3 \\
-3 & 0 & 5 \\
-3 & 5 & 5
\end{array}\right]} \\
& \left.\begin{array}{ccc}
\text { (a) } & \text { (b) } \\
-3 & -3 & 5 \\
-3 & 0 & 5 \\
-3 & -3 & 5
\end{array}\right]\left[\begin{array}{ccc}
-3 & 5 & 5 \\
-3 & 0 & 5 \\
-3 & -3 & -3
\end{array}\right]\left[\begin{array}{ccc}
5 & 5 & 5 \\
-3 & 0 & -3 \\
-3 & -3 & -3
\end{array}\right]\left[\begin{array}{ccc}
5 & 5 & -3 \\
5 & 0 & -3 \\
-3 & -3 & -3
\end{array}\right]
\end{aligned}
$$

(e)

Figure 3. Arrays of kirsch's template in different orientations (a) $0^{\circ}$ (b) $45^{\circ}$ (c) $90^{\circ}$ (d) $135^{\circ}$ (e) $180^{\circ}$ (f) $225^{\circ}(\mathrm{g}) 270^{\circ}$ (h) $315^{\circ}$

\subsection{Morphological Closing and Object Classification}

The method of morphological closing is conducted after the edges have been detected. It is needed to close the holes or empty space within the retina blood vessel [9] and the result is displays in Figure 4 (b). The morphological closing operation is a process of dilation followed by an erosion, using he same structuring element for both operations. Operation of dilation thickens the retina blood vessels in the binary image. The thickening happens because of the controling shape referred as the structuring element. It is a means of interpret the start of the structuring element all through the domain of the image and see whether it overlaps with 1valued pixels [27]. While, erosion is an operation of shrinks or thins in a binary image. It also, controlled by the structuring element as in dilation. The result of structuring element overlaps only 1-valued pixels in the binary image happen when the output of erosion consists of value 1 at each location of the structuring element origin. Mathematically, the morphological closing of A and B is denoted by $\bullet \cdot B$, where:

$$
\mathrm{A} \cdot \mathrm{B}=(\mathrm{A} \oplus \mathrm{B}) \mathrm{B}
$$

geometrically, A $\bullet \mathrm{B}$ is the complement of the union of all translations of B that do not overlap with A.

The process is continued with the object classification. The method is conducted to remove the small object, or noise that is created during the edge detection and morphological closing method. By using bwareaopen in the image processing toolbox in MATLAB, the noises and the unwanted small object is successfully removed. Figure 4 (c) display the image result of successfully removing the noise and unnecessary objects present in the previous image. The function removes all the connected components (objects) present in the binary image that have fewer than threshold pixel and producing another binary image. This operation is known as an area opening. The threshold is the area of unwanted object that need to be removed. It can be set to get the exact result that the user wants. Figure 4 (d) and (e) is the result image of method used by S. Badsha et al. and the ground truths of the input image respectively.

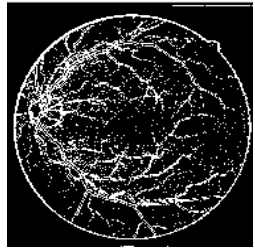

(a)

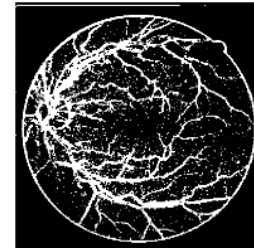

(b)

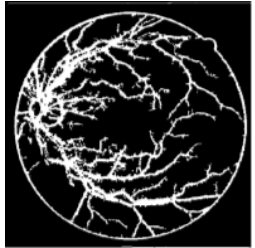

(c)

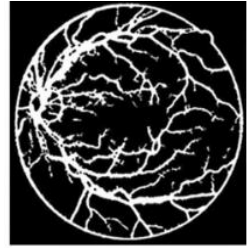

(d)

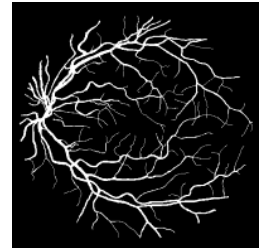

(e)

Figure 4. Feature Extraction (a) Blood vessel extraction image using Kirsch's template (b) morphological closing image (c) After object classification image (d) Blood vessel extraction image by S. Badsha et al. using Kirsch's template (e) groundtruth image. 
Table 1 shows the list of formula to calculate the performance measures. The measure of sensitivity is to determine the ability of the proposed method to successfully detect the vessel pixels. While, the specificity is to compute the proposed method ability to detect the non-vessel pixels. For the accuracy, the ratio between the sum of correctly detected vessels and non-vessels pixels with the total number of pixels is calculated. The total number of false positive (FP), false negative (FN), true positive (TP), and true negative (TN) pixels are used as the parameter for the formula to quantify the extraction performance. The comparison of the images will be between the ground truth image and the segmented image [21].

Table 1. The Performance Measures for Retina Blood Vessel Segmentation

\begin{tabular}{cc}
\hline Measure & Description \\
\hline Sensitivity & $\frac{T P}{(T P+F N)}$ \\
Specificity & $\frac{T N}{(F P+T N)}$ \\
Accuracy & $\frac{T P+T N}{(T P+T N+F P+F N)}$ \\
\hline
\end{tabular}

\section{RESULTS AND ANALYSIS}

The proposed method is evaluated on two online databases as shown in the Figure 5 which are the DRIVE and HRF. The DRIVE database has been conducted to enable comparative studies on segmentation of blood vessels in retinal images. The data in the database were obtained from Netherlands through the program of a diabetic retinopathy screening. The population of screening involve of 400 diabetic patients of age 25-90 years old. The image was acquired using Canon CR5 non-mydriatic 3CCD camera with a 45-degree field of view (FOV) [3].

Next, is the HRF which has been organized by a collaborative research group to support comparative studies on automatic segmentation algorithms on retinal fundus image. The group consist of two European institutions which are the Pattern Recognition Lab (CS5), The Department of Ophthalmology, University Erlangen-Nuremberg (Germany), and the Brno University of Technology, Faculty of Electrical Engineering and Communication, Department of Biomedical Engineering, Brno (Czech Republic). The data is obtained at the collaborating Tomas Kubena's Opthalmology Clinic, Zlin (Czech Republic). The images were captured using a mydriatic fundus camera CANON CF-60 UVi equipped with CANON EOS-20D digital camera with a 60-degree field of view (FOV) [4].

Table 2 shows the result of the MSE and PSNR for the pre-processing part. The closer the value of MSE to zero, the better the result. While, for the PSNR the higher the value, the better the quality of the image. The calculation is conducted to measure the effectiveness of the method used. The results are evaluated on ten images from each database, which 5 of the images is the healthy retina images and another five is the unhealthy retina image. shows that the method has minimum error and the image quality is suitable to continue with further method.

Table 2. The Result of the MSE and PSNR for the Online Databases

\begin{tabular}{|c|c|c|c|c|c|}
\hline \multicolumn{3}{|c|}{ DRIVE } & \multicolumn{3}{|c|}{ HRF } \\
\hline Image & MSE & PSNR & Image & MSE & PSNR \\
\hline \multicolumn{6}{|c|}{ Healthy retina images } \\
\hline Image 1 & 0.0123 & 67.2227 & Image 1 & 0.0181 & 65.5563 \\
\hline Image 2 & 0.0161 & 66.0642 & Image 2 & 0.0165 & 65.9465 \\
\hline Image 3 & 0.0240 & 64.3362 & Image 3 & 0.0280 & 63.6662 \\
\hline Image 4 & 0.0313 & 63.1781 & Image 4 & 0.0352 & 62.6604 \\
\hline Image 5 & 0.0232 & 64.4801 & Image 5 & 0.0369 & 62.4656 \\
\hline \multicolumn{6}{|c|}{ Unhealthy retina images } \\
\hline Image 6 & 0.0187 & 65.4220 & Image 6 & 0.0267 & 63.8667 \\
\hline Image 7 & 0.0239 & 64.3517 & Image 7 & 0.0242 & 64.3013 \\
\hline Image 8 & 0.0267 & 63.8686 & Image 8 & 0.0258 & 64.0223 \\
\hline Image 9 & 0.0260 & 63.9750 & Image 9 & 0.0253 & 64.1022 \\
\hline Image 10 & 0.0289 & 63.5222 & Image 10 & 0.0279 & 63.6737 \\
\hline
\end{tabular}

The result is not only being compared based on the visuality between the segmented image and the groundtruth image, it also evaluates on the performance of the proposed method statistically. Figure 5 displays the result of the proposed method evaluated on the two databases and the method used by S. Badsha et al 
comparing with the ground truth image. While, Table 3 indicates the results of the performance measures evaluated on the two online databases in terms of the accuracy, sensitivity and the specificity. The results evaluated on the DRIVE database gives the accuracy of 0.7597 , sensitivity of 0.7767 and the specificity of 0.7215 . While HRF databases achieves the accuracy of 0.7917 , the sensitivity of 0.9077 and the specificity of 0.5832. The results show that HRF database can detect the vessel and non-vessel pixel more accurately than the DRIVE database. Generally, HRF have more higher image quality that the DRIVE database, which make the image clearer.

(a)
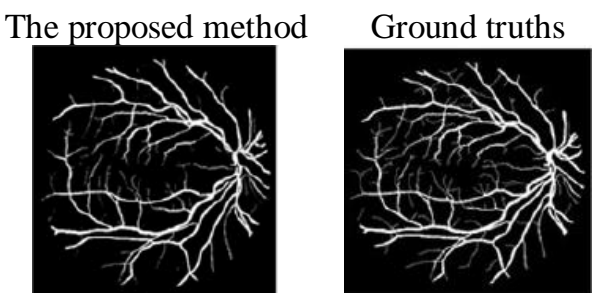

(b)
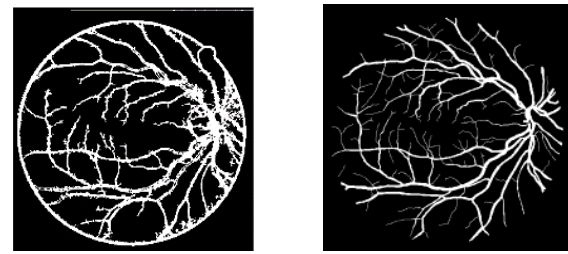

(c)
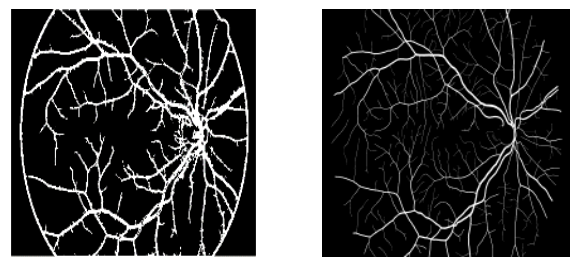

Figure 5. The comparison between the image result of segmentation method and the groundtruths (a) S. Badsha et al. image result using DRIVE (b) The proposed method image result using DRIVE

(c) The proposed method image result using HRF

Table 3. The Performance Measure of DRIVE and HRF Databases

\begin{tabular}{cccc}
\hline Database & Accuracy & Sensitivity & Specificity \\
\hline DRIVE & 0.7597 & 0.7767 & 0.7215 \\
HRF & 0.7917 & 0.9077 & 0.5832 \\
\hline
\end{tabular}

\section{CONCLUSION}

This paper described a method to efficiently extract the retina blood vessel in the fundus images by using the Kirsch template. The DRIVE and HRF databases are used to validate the proposed method. The performance of the method applied is measured in terms of sensitivity, specificity and accuracy between the extract image and the ground truth image to see the effectiveness of the applied method. The results indicate that the proposed algorithm able to perform well at the early stage of detection where unsharp masking, CLAHE and Gaussian filtering is conducted based on the MSE calculation. Then, continue with the kirsch's template to extract the blood vessel, morphological closing to close the empty space in the edges of blood vessel and object classification for the removal of unwanted object to finalize the proposed algorithm. However, the proposed algorithm is lack in detecting the fine vessels accurately due to the noise which has the same size as the fine blood vessels which needed to be removed. For the future work, the proposed method can be improved by undergo further post-processing method.

\section{ACKNOWLEDGEMENTS}

The author would like to thank Research, Innovation, Commercialization and Consultation Management (ORICC) Universiti Tun Hussein Onn Malaysia (H203 Tier 1 Fund) and Registrar Office for sponsoring the research and development of this project. 


\section{REFERENCES}

[1] S. M. Lavin P, Patrylo M, Hollar M, Espaillat KB, Kirshner H, Stroke Risk and Risk Factors in Patients With Central Retinal Artery Occlusion, vol. 196. Elsevier Inc., 2018.

[2] H. Safi, S. Safi, A. Hafezi-Moghadam, and H. Ahmadieh, "Early detection of diabetic retinopathy," Surv. Ophthalmol., vol. 63, pp. 601-608, 2018.

[3] J. Staal et al., "Ridge-based vessel segmentation in color images of the retina," IEEE Trans. Med. Imaging, vol. 23, no. 4, pp. 501-509, 2004.

[4] A. Budai, R. Bock, A. Maier, J. Hornegger, and G. Michelson, "Robust Vessel Segmentation in Fundus Images," Int. J. Biomed. Imaging, vol. 2013, pp. 1-11, 2013.

[5] Z. Yavuz and C. Köse, "Blood Vessel Extraction in Color Retinal Fundus Images with Enhancement Filtering and Unsupervised Classification,” J. Healthc. Eng., vol. 2017, pp. 1-12, 2017.

[6] L. A. Ashame, S. M. Youssef, and S. F. Fayed, "Abnormality Detection in Eye Fundus Retina," 2018 Int. Conf. Comput. Appl., pp. 285-290, 2018.

[7] S. Das, S. Samaddar, and J. Majumdar, "an Automated Graphical User Interface Based System for the Extraction of Retinal Blood Vessels Using Kirsch'S Template,” Int. J. Adv. Comput. Sci. Appl., vol. 6, no. 6, pp. 86-93, 2015.

[8] A. Khatib, K. Shailaja, "Automatic Detection of Retinal Vessels using Kirsch's Templates and Region Growing," Int. J. Sci. Res. Dev., vol. 3, no. 5, pp. 323-326, 2015.

[9] S. Sreng, N. Maneerat, K. Hamamoto, and R. Panjaphongse, "Automated Diabetic Retinopathy Screening System Using Hybrid Simulated Annealing and Ensemble Bagging Classifier,” Appl. Sci., vol. 8, no. 7, p. 1198, 2018.

[10] C. M. Patil and S. Yogesh Kumarn, "Automatic Blood Vessels Segmentation Extraction in Fundus Images for Identification," Int. Conf. Curr. Trends Comput. Electr. Electron. Commun., pp. 389-393, 2017.

[11] S. Badsha, A. W. Reza, and K. G. Tan, "A new blood vessel extraction technique using edge enhancement and object classification," Soc. Imaging Informatics Med., pp. 1107-1115, 2013.

[12] M. Sivakumar, T. S. Subashini, and G. Sivaranjani, "Exudates Identification and Classification using Kirsch Template and K-means Clustering in Fundus Eye Images,” Int. J. Sci. Res. Comput. Sci. Eng. Inf. Technol., vol. 3, no. 3, pp. 1796-1803, 2018.

[13] R. Geetharamani, L. Balasubramanian, "Retinal blood vessel segmentation employing image processing and data mining techniques for computerized retinal image analysis," Biocybern. Biomed. Eng., 36(1), pp. 102-118, 2016.

[14] R. Kolar et al., "Retinal vessel segmentation by improved matched filtering: evaluation on a new high-resolution fundus image database," IET Image Process., vol. 7, no. 4, pp. 373-383, 2013.

[15] T. Chakraborti, D. K. Jha, A. S. Chowdhury, and X. Jiang, "A self-adaptive matched filter for retinal blood vessel detection," Mach. Vis. Appl., vol. 26, no. 1, pp. 55-68, 2014.

[16] A. Nazari, M. Mustafa, and A. Zulkifley, "Segmentation of retinal blood vessels by top hat multi-scale detection for optic disc removal Article,” J. Teknol., no. November, pp. 49-53, 2015.

[17] U. T. V. Nguyen, A. Bhuiyan, L. A. F. Park, and K. Ramamohanarao, "An effective retinal blood vessel segmentation method using multi-scale line detection," Pattern Recognit., vol. 46, no. 3, pp. 703-715, 2013.

[18] Y. Hou, "Automatic segmentation of retinal blood vessels based on improved multiscale line detection," J. Comput. Sci. Eng., vol. 8, no. 2, pp. 119-128, 2014.

[19] J. Dash and N. Bhoi, "A thresholding based technique to extract retinal blood vessels from fundus images," Futur. Comput. Informatics J., pp. 1-7, 2017.

[20] E. Imani, M. Javidi, and H. R. Pourreza, "Improvement of retinal blood vessel detection using morphological component analysis," Comput. Methods Programs Biomed., vol. 118, no. 3, pp. 263-279, 2015.

[21] A. Ali, W. M. D. W. Zaki, and A. Hussain, "Retinal blood vessel segmentation from retinal image using B-COSFIRE and adaptive thresholding,” Indones. J. Electr. Eng. Comput. Sci., vol. 13, no. 3, pp. 1199-1207, 2019.

[22] N. Memari, A. R. Ramli, M. I. Bin Saripan, S. Mashohor, and M. Moghbel, "Supervised retinal vessel segmentation from color fundus images based on matched filtering and AdaBoost classifier," PLoS One, 2017.

[23] D. J. Bora, "Importance of image enhancement techniques in color image segmentation: a comprehensive and comparative study," Indian J.Sci.Res., vol. 15, no. 1, pp. 115-131, 2017.

[24] J. N. Archana and P. Aishwarya, "A review on the image sharpening algorithms using unsharp masking," Int. J. Eng. Sci. Comput., vol. 6, no. 7, pp. 8729-8733, 2016.

[25] B. H. Shekar and K. P. Uma, "Kirsch directional derivatives based shot boundary detection : an e ffi cient and accurate method," Procedia - Procedia Comput. Sci., vol. 58, pp. 565-571, 2015.

[26] H. S. Bhadauria, S. S. Bisht, and A. Singh, "Vessels extraction from retinal images," IOSR J. Electron. Commun. Eng., vol. 6, no. 3, pp. 79-82, 2013.

[27] R. Rodrigo, “Morphological Image Processing,” pp. 1-12, 2009. 


\section{BIOGRAPHIES OF AUTHORS}
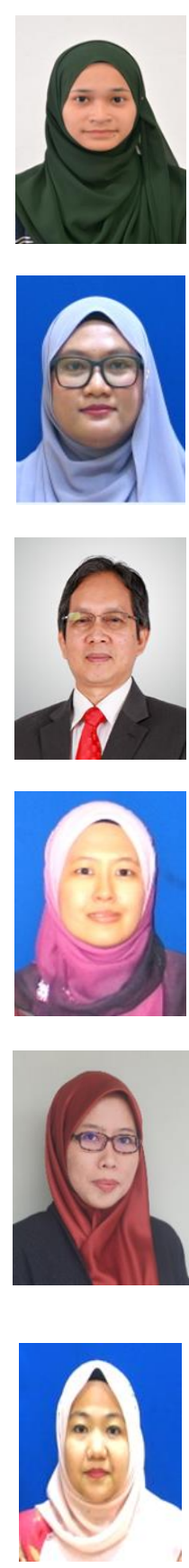

Nur Syazlin Zolkifli was born on $13^{\text {th }}$ January 1996 in Ipoh, Perak. She is currently pursuing degree in electronic engineering with specialization medical at Universiti Tun Hussein Onn Malaysia in Batu pahat, Johor.Her final year project interest are in image processing specialize in medical image. She is an active member of IEM

Ain Nazari received Diploma and B. Eng degree in Electrical Engineering in 2005 and 2008 from Universiti Tun Hussein Onn Malaysia (UTHM), M. Eng (Mechatronics and Automatic Control) and $\mathrm{PhD}$ in Electronics and Computer System in 2010 and 2018 from Universiti Teknologi Malaysia (UTM) and Universiti Kebangsaan Malaysia (UKM), respectively. In 2008 she joined UTHM and is currently a lecturer in the Faculty of Electrical and Electronic Engineering. Her main research interests are in image processing specialize in medical image and computer vision.

Mohd Marzuki Mustafa received the B.Eng. degree in Electrical Engineering in 1985, and the M.Sc. and PhD. Degrees in Contro Systems Engineering in 1986 and 1989 from University of Manchester Institute of Science and Technology, United Kingdom, and University of Salford, United Kingdom, respectively. In 1984, he joined the Universiti kebangsaan Malaysia and is currently a professor in the Faculty of Engineering and Built Environment. His main research interests are in computer control systems \& Instrumentation and Machine vision.

Wan NurShazwani Wan Zakaria received B.Eng. (2007) in Electronics and Mechanical Engineering from Chiba University and MSc in Mechatronics (2008) and PhD in Mechanical and System Engineering (2012) from Newcastle University. She is currently a lecturer in Faculty of Electrical and Electronic Engineering, Universiti Tun Hussein Onn Malaysia. Her current interests include Medical Robotics System specifically on development of robot force control, Image Processing and Computer Aided Diagnosis, and development of Wearable Device. She is author and co-author of several journal papers and conference proceedings.

Nor Surayahani Suriani is Senior Lecturer in Universiti Tun Hussein Onn Malaysia (UTHM). She received her $\mathrm{PhD}$ in Electronics and Computer System from Universiti Kebangsaan Malaysia (UKM) in 2015 and finished her Master (Universiti Teknologi Malaysia) and Bach. Eng. (Universiti Putra Malaysia) in Electronics and Communication in 2007 and 2003, respectively. Her research interest focuses on computer vision, deep learning, image processing and bioinspired visual cortex algorithm development. She has published in Q1 impact factor and Scopus journals mainly in image processing and computer vision areas. She also a member in national Board of Engineers.

Wan Nur Hafsha Wan Kairuddin received her M.Eng. (Electrical Engineering) from Universiti Tun Hussein Onn Malaysia (UTHM) in 2016, and B.Eng. (Electronics Engineering) in 2005 from Universiti Sains Malaysia (USM). She is currently an Instructor Engineer in Universiti Tun Hussein Onn Malaysia (UTHM). Her research interest focuses on medical image processing and healthcare system development. She received certification as Professional Technologist in 2019 from Malaysia Board of Technologies. She also a member of national Board of Engineers. 